\title{
Migraine misdiagnosis as a sinusitis, a delay that can last for many years
}

\author{
Jasem Y Al-Hashel ${ }^{1,2}$, Samar Farouk Ahmed ${ }^{1,3^{*}}$, Raed Alroughani ${ }^{4,5}$ and Peter J Goadsby ${ }^{6}$
}

\begin{abstract}
Background: Sinusitis is the most frequent misdiagnosis given to patients with migraine. Therefore we decided to estimate the frequency of misdiagnosis of sinusitis among migraine patients.

Methods: The study included migraine patients with a past history of sinusitis. All included cases fulfilled the International Classification of Headache Disorders, $3^{\text {rd }}$ edition (ICHD-III- beta) criteria. We excluded patients with evidence of sinusitis within the past 6 months of evaluation. Demographic data, headache history, medical consultation, and medication intake for headache and effectiveness of therapy before and after diagnosis were collected.
\end{abstract}

Results: A total of 130 migraine patients were recruited. Of these patients $106(81.5 \%)$ were misdiagnosed as sinusitis. The mean time delay of migraine diagnosis was ( $7.75 \pm 6.29$, range 1 to 38 years). Chronic migraine was significantly higher $(p<0.02)$ in misdiagnosed patients than in patients with proper diagnosis. Medication overuse headache $(\mathrm{MOH})$ was reported only in patients misdiagnosed as sinusitis. The misdiagnosed patients were treated either medically $87.7 \%$, or surgically $12.3 \%$ without relieve of their symptoms in $84.9 \%$ and $76.9 \%$ respectively. However, migraine headache improved in 68.9\% after proper diagnosis and treatment.

Conclusions: Many migraine patients were misdiagnosed as sinusitis. Strict adherence to the diagnostic criteria will prevent the delay in migraine diagnosis and help to prevent chronification of the headache and possible $\mathrm{MOH}$.

Keywords: Migraine misdiagnosis; Sinus headache

\section{Background}

Migraine continues being an underdiagnosed condition [1] because it can be accompanied by symptoms commonly associated with other causes of facial pain $[2,3]$. Many patients visit their general practitioner because of their headache and, in many cases, a proper diagnosis and treatment may take years $[4,5]$.

"Sinusitis" may constitute one of the most commonly confusing clinical presentation of migraine [6], probably because cranial autonomic symptoms are common in migraine [7] based on activation of the trigeminal-autonomic reflex [8]. Headaches located in the frontal, supraorbital, or infraorbital region are sinus headaches [9]. These headaches are usually recurrent, non-seasonal, and unassociated with fever, localized tenderness, or erythema [10].

\footnotetext{
* Correspondence: samerelshayb@hotmail.com

'Department of Neurology, Ibn Sina Hospital, P.O. Box 25427, Safat 13115 Kuwait City, Kuwait

${ }^{3}$ Department of Neurology and Psychiatry, Al-Minia University, Minia, Egypt Full list of author information is available at the end of the article
}

Migraine-associated alterations in trigeminal and/or autonomic activity may explain nasal and ocular symptoms in migraine. For example, "sinus" symptoms in migraine have been hypothesized to arise from activation of the trigeminal-autonomic reflex, which is mediated by a circuit of trigeminal afferents and parasympathetic efferent that innervate the lacrimal glands and the nasal mucosa [11].

This study aimed to estimate the frequency of misdiagnosis of sinusitis among patients with migraine headache who fulfilled the diagnostic criteria according to the International Classification of Headache Disorders, $3^{\text {rd }}$ edition (ICHD-III- beta) criteria [12].

\section{Methods}

This retrospective study included 130 male and female migraine patients aged above 12 years with history of sinusitis. Every headache was assigned a diagnosis based ICHD-IIIbeta [12].

\section{实}


Exclusion criteria included radiographic evidence of sinus infection, the occurrence of fever, or purulent nasal discharge associated with their headaches within the past six months of evaluation. Patients who were unable to give reliable information about their medical history and headache characteristics or have incomplete medical files were excluded from the study $(\mathrm{n}=17)$.

\section{Patient identification}

The data were collected from a hospital-based cohort of headache patients referred to both Mubarak and Ibn Sina Hospitals, Kuwait. We examined the medical files of all patients diagnosed with headache who were registered between 2010 and 2012. The records were examined and standardized data collection forms were completed retrospectively by the study group.

\section{Clinical data}

The diagnosis of migraine was made during face to face interview with headache specialist. Demographic characteristics, headache frequency, duration, and associated headache symptoms were recorded for each patient. Patients were asked about the onset of their headache, how long it took them to receive a correct diagnosis (latency of diagnosis) and what different physicians they had consulted prior to the current consultation. Results of previous diagnostic investigation including brain and sinus imaging were retrieved also.

Patients were asked about their use of medical and surgical treatments for headache before migraine diagnosis and to rate the effectiveness of each treatment before and after diagnosis on a 4-point scale: [13]

1. Very effective - complete and long-lasting relief

2. Effective - partial and/or short-lasting relief

3. Ineffective

4. Headache worsened.

\section{Data analysis}

All analyses were performed using SPSS 19 for Windows. Simple descriptive statistical tests (Mean and Standard deviation) were used to describe the numerical values of the sample. Frequency and percentage were used to describe the non-numerical values of the sample. The significance of the differences between the patients with proper and misdiagnosis was determined using a chi-squared test for non- numerical variables. $P<0.05$ was defined as statistically significant.

The study received the approval of the local ethic committee, and all the patients signed the appropriate informed consents.

\section{Results}

Table 1 describes the demographic and characteristic data of 130 migraine patients.

\section{Symptoms}

The symptoms referred to the sinus areas were: sinus pain (76.2\%), sinus pressure (60\%) and nasal congestion (55.4\%). Most of our patients had at least one investigation looking at the sinuses. Thirteen patients $(10 \%)$ showed thickened sinus mucosa in CT sinuses. Many patients had at least one performed neuroimaging test and all of them were normal (Table 1).

\section{Headache diagnosis}

We found that 106 (81.5\%) of our patients had been misdiagnosed as sinusitis. Chronic migraine was significantly higher $(p<0.0001)$ in misdiagnosed patients and medication over use headache $(\mathrm{MOH})$ reported only in patients misdiagnosed as sinusitis (Table 2).

\section{Misdiagnosis results}

The mean duration of headache in misdiagnosed patients was $11.15 \pm 7.85$ (range 2 to 40 years) and the mean time between the first attack of headache and the diagnosis of migraine was $7.75 \pm 6.29$ (range 1 to 38 years). 59/106 (56.6\%) of them had consulted a primary care physician, and $47 / 106$ patients (43.4\%) assessed by otorhinolaryngology specialist before the diagnosis of migraine was made.

Thirteen patients $(12.3 \%)$ of them had prior "sinus surgery" based on suspected lesions on CT sinuses and 93

Table 1 Demographic data and characteristic of all migraine patients $(n=130)$ according to ICHD-III-beta

\begin{tabular}{ll}
\hline Variable & Mean \pm SD/no. (\%) \\
\hline Age & $35.88 \pm 9.87$ \\
Duration of headache & $10.22 \pm 7.60$ \\
Sex & \\
Male & $30(23.1 \%)$ \\
Female & $100(76.9 \%)$ \\
Symptoms referred to sinus area & \\
Sinus pain & $99(76.2 \%)$ \\
Sinus congestion & $78(60 \%)$ \\
Nasal congestion & $72(55.4 \%)$ \\
Investigations looking at the sinuses & \\
Sinus $x$ ray & $58(44.6 \%)$ \\
Sinus CT & $21(16.2 \%)$ \\
Endoscopy & $3(2.3 \%)$ \\
Neuroimaging & \\
MRI brain & $44(33.8 \%)$ \\
$C T$ brain & $6(4.6 \%)$ \\
MRI cervical spine & $2(1.5 \%)$ \\
\hline
\end{tabular}


Table 2 Comparison of headache profiles according to ICHD-III between migraine patients with proper diagnosis and those with misdiagnosis

\begin{tabular}{llll}
\hline Type of headache & $\begin{array}{l}\text { Migraine patients with } \\
\text { proper diagnosis }(\mathbf{n = 2 4 )}\end{array}$ & $\begin{array}{l}\text { Migraine patients misdiagnosed } \\
\text { as sinusitis }(\mathbf{n}=\mathbf{1 0 6})\end{array}$ \\
\hline Migraine without aura [1.1] & $13(54.2 \%)$ & $43(40.6 \%)$ & P value \\
Migraine with aura [1.2] & $8(33.3 \%)$ & $22(20.8 \%)$ & 0.2 \\
Chronic migraine [1.3] & $3(12.5 \%)$ & $30(28.3 \%)$ & 0.1 \\
Medication over use headache [8.2] & 0 & $11(11.4 \%)$ & $0.02^{*}$ \\
\hline
\end{tabular}

*Significant.

(87.7\%) received medical treatment for sinusitis. Both surgical and medical treatment were ineffective in most of the patients. Of those who received ineffective medical or surgical treatment, 73 (69\%) were more satisfied in 24 months after initiating anti-migraine treatment (topiramate, propranolol, amitriptyline or sodium valproate) after their proper diagnosis (Table 3).

\section{Discussion}

Our study included 130 patients with migraine-type headache according to $3^{\text {rd }}$ edition (ICHD-III-beta). We found that $81.5 \%$ of them were misdiagnosed and managed as sinusitis. The similarity of sinusitis symptoms and migraine complicates the diagnostic evaluation process. Although both historical and new data show that nasal symptoms frequently accompany a migraine, these symptoms are not required by the ICHD-III-beta diagnostic criteria for a migraine.

Our data are in agreement with the study of Schreiber and colleagues [6] which included approximately 3000

\begin{tabular}{|c|c|}
\hline Variable & No. (\%) \\
\hline \multicolumn{2}{|l|}{ Effective of management prior migraine diagnosis } \\
\hline \multicolumn{2}{|l|}{ Surgical $(\mathrm{no}=13)$} \\
\hline Very effective - complete and long-lasting relief & $0 / 13$ \\
\hline Effective -partial and/or short-lasting relief & $3 / 13(22.1 \%)$ \\
\hline Ineffective & $9 / 13(69.2 \%)$ \\
\hline Headache worsened & $1 / 13(7.7 \%)$ \\
\hline \multicolumn{2}{|l|}{ Medical $(\mathrm{no}=93)$} \\
\hline Very effective - complete and long-lasting relief & $0 / 93$ \\
\hline Effective - partial and/or short-lasting relief & 14/93 (15.1\%) \\
\hline Ineffective & $65 / 93(69.8 \%)$ \\
\hline Headache worsened & 14/93 (15.1\%) \\
\hline \multicolumn{2}{|l|}{$\begin{array}{l}\text { Effective of treatment after migraine } \\
\text { diagnosis }(n o=106)\end{array}$} \\
\hline Very effective - complete and long-lasting relief & $73 / 106(68.9 \%)$ \\
\hline Effective -partial and/or short-lasting relief & 22/106 (20.8\%) \\
\hline Ineffective & $8 / 106(7.5 \%)$ \\
\hline Headache worsened & $3 / 106(2.8 \%)$ \\
\hline
\end{tabular}

patients with a history of self-described or physiciandiagnosed "sinus" headache and they determined that $80 \%$ of patients met ICHD criteria for migraine. Our data are also in agreement with the other previous studies $[3,9,14,15]$ which reported that "sinus headache" is one of the most commonly reported terms used in combination with a migraine diagnosis and most patients presenting with a "sinus headache" may not actually have a rhino sinusitis associated headache.

Migraine can be mistaken for rhinosinusitis because of similarity in location of the headache and the commonly accompanying nasal autonomic symptoms. The presence or absence of purulent nasal discharge and/or other features diagnostic of acute rhinosinusitis help to differentiate these conditions [12]. In order to properly establish a diagnosis of migraine, it is essential to know the ICHD criteria and apply these criteria in clinical practice.

We demonstrated that chronic migraine was significantly higher in patients misdiagnosed with sinusitis. $\mathrm{MOH}$ was reported only in those patients. A delay in the diagnosis of migraine led to chronification of the headache and transformation, in some cases, into $\mathrm{MOH}$.

We found that the diagnosis of migraine was delayed in more than $80 \%$ of our cohort up to 38 years. Eross and colleagues [15] similarly found that their patients waited 25.3 years (longest of 62 years) prior to the correct diagnosis. Previous studies showed this as well $[4,5]$. The diagnostic delay in our cohort could be explained by the presence of sinus pain, sinus congestion and nasal discharge during headache attacks. These symptoms have been reported with previous studies which concluded that presence of autonomic symptoms during migraine attacks often leads to confusion and incorrect diagnosis of sinusitis $[16,17]$. The ICHD criteria do not highlight the presence of cranial autonomic symptoms in the disorder, or perhaps more usefully comment upon them. This may help general practitioner and otorhinolaryngology specialist to be aware of the phenotyping overlap.

The majority of our patients had at least one investigation looking at the sinuses which were all normal. This result is similar to previous results which demonstrated that patients with "sinus headache" did not have findings suggestive of sinusitis on endoscopy or CT scan [14] and over $50 \%$ of them were diagnosed with migraine later [18]. 
These unnecessary investigations increase time delay to obtain the correct diagnosis and management [19].

We demonstrated that $56 \%$ of the misdiagnosed patients had consulted a primary care physician and $44 \%$ of them an otorhinolaryngology specialist before the diagnosis of migraine was made. We are in agreement with Foroughipour and colleagues [17] who studied 58 patients with the diagnosis of sinusitis made by a primary care physician. After comprehensive otorhinolaryngologic and neurologic evaluation, the final diagnoses was migraine in $68 \%$ of the patients. Furthermore, our study demonstrated that the misdiagnosed patient received either medical in $87.7 \%$, or surgical treatment in $12.3 \%$ of them without relieve of their symptoms in $84.9 \%$ and $76.9 \%$ respectively. However, migraine headache improved in $68.9 \%$ after proper diagnosis and treatment. These results are similar to that of Foroughipour and colleagues [17] who reported that recurrent antibiotic therapy was received by $66 \%$ patients and therapeutic nasal septoplasty was performed in $16 \%$ of the patients with a final diagnosis of migraine.

An appropriate recognition of migraine in patients who complain about sinus headaches may help to minimize the suffering and unnecessary interventions, start migraine directed therapy [20] and improve quality of life [9].

\section{Conclusion}

In conclusion, symptoms suggestive of sinusitis are frequently seen in migraine patients and may lead to delayed diagnosis and treatment of migraine. General practitioner and otorhinolaryngology specialist should be aware of the diagnostic criteria for migraine and consider it in their differential diagnosis of patients suffering from "sinusitis". Going forward it is important to consider how best to draw attention to cranial autonomic symptoms in migraine and their place in diagnostic criteria.

\section{Competing interest}

The authors declare that there are no conflicts of interest.

\section{Authors' contributions}

JAH designed the study, collected the data and revised the manuscript, SFA collected, and analyzed the data, and wrote the manuscript, RA and PJG revised the manuscript. All authors read and approved the final manuscript.

\section{Funding}

This research received no specific grant from any funding agency in the public, commercial, or not-for-profit sectors.

\section{Author details}

'Department of Neurology, Ibn Sina Hospital, P.O. Box 25427, Safat 13115 Kuwait City, Kuwait. ${ }^{2}$ Department of Medicine, Faculty of Medicine, Health Sciences Centre, Kuwait University, Kuwait City, Kuwait. ${ }^{3}$ Department of Neurology and Psychiatry, Al-Minia University, Minia, Egypt. ${ }^{4}$ Division of Neurology, Amiri Hospital, P.O. Box 1661, Qurtoba 73767 Kuwait City, Kuwait. ${ }^{5}$ Division of Neurology, Dasman Diabetes Institute, P.O. Box 1180, Dasman 15462 Kuwait City, Kuwait. ${ }^{6}$ Wellcome Trust Clinical Research Facility, Kings College Hospital, London, UK.
Received: 28 September 2013 Accepted: 2 December 2013

Published: 12 December 2013

\section{References}

1. Stewart WF, Simon D, Shechter A, Lipton RB (1995) Population variation in migraine prevalence: a meta-analysis. J Clin Epidemiol 48:269-80

2. Cady RK, Schreiber CP (2002) Sinus headache or migraine? considerations in making a differential diagnosis. Neurology 58(suppl 6):S10-S14

3. Diamond ML (2002) The role of concomitant headache types and nonheadache comorbidities in the underdiagnosis of migraine. Neurology 58(suppl 6):S3-S9

4. De Diego EV, Lanteri-Minet M (2005) Recognition and management of migraine in primary care: influence of functional impact measured by the headache impact test (HIT). Cephalalgia 25(3):184-90

5. Ryan RE, Jr, Pearlman SH (2004) Common headache misdiagnoses. Prim Care Clin Office Pract 31:395-405

6. BCPS; Powers C, Schreiber CP, Hutchinson S, Webster CJ, Ames M, Richardson MS, Pharm D (2004) Prevalence of migraine in patients with a history of self-reported or physician-diagnosed "Sinus" headache. Arch Intern Med 164:1769-1772

7. Peter JG (2009) Lacrimation, conjunctival injection, nasal symptoms...cluster headache, migraine and cranial autonomic symptoms in primary headache disorders- what's new? J Neurol Neursurg Psychiatry 80:1057-1058

8. May A, Goadsby PJ (1999) The trigeminovascular system in humans: pathophysiological implications for primary headache syndromes of the neural influences on the cerebral circulation. J Cereb Blood Flow Metab 19:115-127

9. Dadgarnia MH, Atighechi S, Baradaranfar MH (2010) The response to sodium valproate of patients with sinus headaches with normal endoscopic and CT Findings. Eur Arch Otorhinolaryngol 267:375-379

10. Levine HL, Setzen M, Cady RK, et al. (2006) An otolaryngology, neurology, allergy and primary care consensus on diagnosis and treatment of sinus headache. A literature review. Otolaryngol Head Neck Surg 134:516-523

11. Akerman S, Holland P, Goadsby PJ (2011) Diencephalic and brainstem mechanisms in migraine. Nat Rev Neurosci 12:570-584

12. Headache Classification Committee of the International Headache Society (2013) The International Classification of Headache Disorders, 3rd edition (beta version). Cephalalgia 33:629-808

13. Rossi P, Faroni J, Tassorelli C, Nappi G (2009) Diagnostic delay and suboptimal management in a referral population with hemicrania continua. Headache 49:227-234

14. Mehle ME, Kremer PS (2008) Sinus CT scan findings in "Sinus Headache" migraineurs. Headache 48:67-71

15. Eross E, Dodick D, Eross M (2007) The Sinus, Allergy and Migraine Study (SAMS). Headache 47:213-224

16. Barbanti P, Fabbrini G, Pesare M, et al. (2002) Unilateral cranial autonomic symptoms in migraine. Cephalalgia 22:256-259

17. Foroughipour M, Sharifian SM, Shoeibi A, Ebdali Barabad N, Bakhshaee M (2011) Causes of headache in patients with a primary diagnosis of sinus headache. Eur Arch Otorhinolaryngol 268(11):1593-6

18. Perry BF, Login IS, Kountakis SE (2004) Nonrhinologic headache in atertiary rhinology practice. Otolaryngol Head Neck Surg 130:449-452

19. Viticchi G, Silvestrini M, Falsetti L, Lanciotti C, Cerqua R, Luzzi S, Provinciali L, Bartolini M (2011) The role of instrumental examinations in delayed migraine diagnosis. Neurol Sci 32(Suppl 1):S143-4

20. Patel ZM, Kennedy DW, Setzen M, Poetker DM, Delgaudio JM (2013) "Sinus headache": rhinogenic headache or migraine? An evidence-based guide to diagnosis and treatment. Int Forum Allergy Rhinol 3:221-230

doi:10.1186/1129-2377-14-97

Cite this article as: Al-Hashel et al:: Migraine misdiagnosis as a sinusitis, a delay that can last for many years. The Journal of Headache and Pain 2013 14:97. 